\title{
HELIOSEISMOLOGICAL DETERMINATION OF STRATIFICATION AND DYNAMIC PROCESSES IN THE SOLAR CORE
}

\author{
A.G. KOSOVICHEV \\ Crimean Astrophysical Observatory \\ 334413, Nauchny, Crimea, USSR
}

Helioseismology provides a powerful tool for investigating stratification and dynamics of plasma in the sun's interior. The observed frequencies of the 5-min oscillations depend on density, sound speed, chemical abundances and angular velocity distributions inside the Sun. These parameters are estimated using inversion techniques.

The inversion of the irequencies presented by Duvall et al. (1988) gives some evidence for material redistribution in the energy-generating core as compared with a standard solar model (Gough \& Kosovichev, 1989). The helioseismological inversion results lead to conclusions that the evolutionary age of the Sun is perhaps 10 per cent greater than it is generally accepted and the neutrino flux from ${ }_{B} B$ decay may be about 60 per cent of the standard model value as a result of mixing. Outside the solar core the sound speed is about 1 per cent greater than it is in the standard solar model. The result is evidence that the energy transport is less effective in the layers between 0.4 and $0.7 R$ (ChristensenDalsgaard, 1988).

The rotational frequency splitting data of Duvall and Harvey (1984) and Brown and Morrow (1987) have been analyzed to determine the angular velocity in the sun as a parametric function of radius and latitude using the asymptotic inversion technique (Kosovichev, 1988). The results show that the equatorial rotation rate slowly decreases below the photosphere. The main changes in rotation take place near the bottom of the convective zone where the solar dynamo might operate and in the solar core. The latitudinal differential rotation is close to the surface rotation in the convective zone for $r / R>0.7$ and decreases rapidly in a region below the convective zone for $0.4<r / R<0.7$. The decrease of the latitudinal differential rotation might be connected with a Kelvin-Helmholtz instability (Dziembowski \& Kosovichev, 1987).

\section{REFERENCES}

Brown, T.M. \& Morrow, C.A. 1987. Astrophys. J. Lett., 314, L21. Christensen-Dalsgaard, J. 1988, Seismology of the Sun \& sun-like stars, ESA Publ. SP-286, p. 431.

Duvall Jr, T.L. \& Harvey, J.W. 1984. Nature, 310, 19. Duvall Jr, T.L. et al. 1988. Astrophys. J., 324, 1158. Gough, D.O. \& Kosovichev, A.G. 1989, Inside the Sun. (in press). Dziembowski, W. \& Kosovichev, A.G. 1987. Acta Astron., 37, 341. Kosovichev, A.G. 1988. Sov. Astron. Lett., $14,145$. 